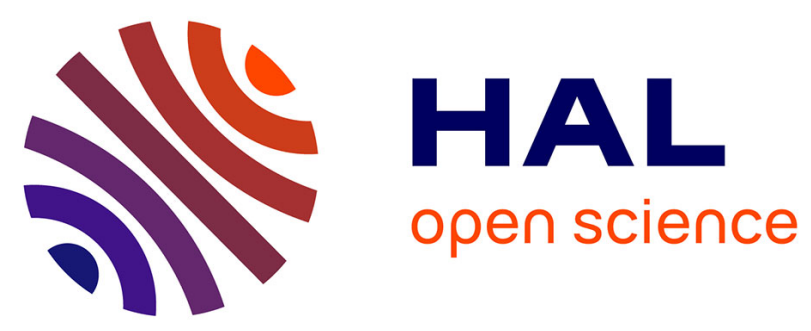

\title{
Time Reversal Applied to Multi-Carrier Reflectometry for On-line Diagnosis in Complex Wiring Systems
}

\author{
Wafa Ben Hassen, Moussa Kafal, Esteban Cabanillas
}

\section{To cite this version:}

Wafa Ben Hassen, Moussa Kafal, Esteban Cabanillas. Time Reversal Applied to Multi-Carrier Reflectometry for On-line Diagnosis in Complex Wiring Systems. 2018 International Automatic Testing Conference (AUTOTESTCON), IEEE, Sep 2018, National Harbor, United States. pp.8532546, 10.1109/AUTEST.2018.8532546 . cea-03049309

HAL Id: cea-03049309 https://hal-cea.archives-ouvertes.fr/cea-03049309

Submitted on 9 Dec 2020

HAL is a multi-disciplinary open access archive for the deposit and dissemination of scientific research documents, whether they are published or not. The documents may come from teaching and research institutions in France or abroad, or from public or private research centers.
L'archive ouverte pluridisciplinaire HAL, est destinée au dépôt et à la diffusion de documents scientifiques de niveau recherche, publiés ou non, émanant des établissements d'enseignement et de recherche français ou étrangers, des laboratoires publics ou privés. 


\title{
Time Reversal Applied to Multi-Carrier Reflectometry for On-line Diagnosis in Complex Wiring Systems
}

\author{
Wafa BEN HASSEN*, Moussa KAFAL*, Member, IEEE, and Esteban CABANILLAS \\ ${ }^{*}$ CEA, LIST, Laboratoire de Fiabilité et Intégration Capteurs, 91191 Gif-sur-Yvette, France. \\ moussa.kafal@cea.fr
}

\begin{abstract}
In wiring networks, on-line diagnosis aims at detecting and locating faults in live cables concurrently to their normal operation. Within this context, Multi-carrier Reflectometry (MCR), a reflectometry based method, not only succeeded in this mission but also permitted controlling the signal bandwidth and thus avoiding false alarms. However, it has shown to suffer from signal loss related to wiring system's attenuation, coupling and network-topology complexity. On the other hand, Time Reversal (TR) signal processing recently adapted with efficiency to wire diagnosis, has proven to boost the performance with an increased network response's complexity accompanied with a higher detection gain. In this paper, we propose a fusion between TR and MCR in a TR Multi-Carrier Reflectometry (TRMCR) method aiming at maximizing the coverage of the online diagnosis. Simulation and experimental results demonstrate that TRMCR permits to increase the peak's signature at detected impedance discontinuities in a network under test (NUT) when compared to what the standard MCR produce.
\end{abstract}

Index Terms-On-line Cable Diagnosis; Wire Networks, Fault detection and location, Multi-Carrier Reflectometry; Time reversal.

\section{INTRODUCTION}

In many application domains, faults affecting wire networks can suddenly occur and can lead to dramatic consequences. Accordingly, live or embedded wire diagnosis has recently attracted the curiosity of researchers since it is often essential to ensure permanent health monitoring of embedded cables during their normal operation. This implies additional constraints related to the diagnosis harmlessness. In fact, testing signals must not interfere with the useful signals. To do so, the choice of the injected signals must be judicious to avoid the frequency bands used by the target system which is often referred to as the prohibited bandwidth. This permits evenly to detect intermittent faults that seem to be brief, but may be sometimes destructive such as electric arcs. However, the presence of useful signals of the target system could affect the test signals due to interference, coupling and limited bandwidth constraints.

Several variants of reflectometry methods, which are techniques basically relying on the injection of a testing signal into the NUT and monitoring the reflected ones by impedance discontinuities (IDis) [1], have been proposed to bypass the aforementioned problems and improve the on-line diagnosis of embedded NUTs. Notably, the idea of linking time domain reflectometry (TDR) and spread spectrum techniques led to novel methods using pseudo random binary sequences as test signals, which were referred to as the Sequence Time Domain
Reflectometry (STDR) [2] and the Spread Spectrum TDR (SSTDR) [1]. In literature, SSTDR makes it possible to shift the spectrum of the transmitted signal from the forbidden band (operating band of the system) through the application of carrier frequency modulation of the pseudo-random bit sequences of the SSTDR. However the spectral occupancy is twice as large as the STDR reflectometry and the spectrum can just be shifted but not controlled entirely.

Recently, Multi-Carrier-based reflectometry methods (MCR) [3]-[5] showed very good potential for the diagnosis of live wire networks by taking benefit of the multi-carrier signals' capacity to control the signal bandwidth. Within the same context, a new method called Orthogonal Multi-tone Time Domain Reflectometry (OMTDR) has been proposed [6]. It applies the principles of Orthogonal Frequency Division Multiplexing (OFDM) to wired network diagnosis. The idea is to divide the bandwidth into multiple sub-bands using orthogonal and then overlapped sub-carriers which permits to maximize the spectral efficiency and the total spectrum control. Accordingly, the prohibited frequency band may be avoided by canceling the corresponding tone of the OMTDR signal. As a matter of fact, MCR and its variant OMTDR have shown promising and efficient potential in detecting and locating hard and soft faults in an online live manner. However, they suffered from signal loss related to wiring system's attenuation, coupling and complex wiring topology.

In another aspect, the problem of fault location in a complex wire network is closely related to the problem of target location encountered in radar detection where increasing multiple scattering in the medium poses a significant difficulty when the target's signature is weak compared with other scatterers. To deal with this difficulty, time-reversal techniques (TR) [7], [8] can ensure a maximization of the fault-related echo by maximizing the energy impinging on the fault position with respect to the rest of the system under test. This idea was transposed to guided-wave propagation, e.g., wire networks, in [9]-[13] and has shown to precisely detect and locate single as well as multiple soft faults even in complex network configurations, involving a number of junctions. In fact, the complexity of the NUT has proven to positively enhance the performance of the TR methods, as an increasingly longer sequence of echoes systematically leads to a higher fraction of energy being concentrated into the fault-related peak echo.

In this paper, we propose applying TR to the MCR as a TRMCR method aiming at maximizing the coverage of the on- 
line diagnosis. To do so, the multi-carrier signal is generated and injected down the NUT. During its propagation, part of its energy is reflected back to the injection point at each IDis (junction, fault, connector, etc.). The reflected echoes are recorded, time reversed and numerically re-injected into a cloned numerical model of the NUT. The IDis locations can be inferred by correlating the new TR reflected echoes to the incident TR signal. Simulation tests were conducted on a point-to-point single branch cable with a capacitive fault in addition to a complex wire network composed of 11 branches and 5 junctions. For both NUTs, TRMCR has increased the faults and junctions signatures with a factor of 2 compared to OMTDR test results. For an additional feasibility proof, experimental tests were applied on a $35 \mathrm{~m}$ long STP EN 2714013 aeronautical MLB cable with a D38999 connector placed at $30 \mathrm{~m}$. An electronic board including a FPGA SoC Xilinx ZyNQ 7010, 10-bits ADC, 10-bits DAC and 512 Megabyte DDR was used to generate, inject and receive the multicarrier OFDM signals. Significantly, TRMCR not only located accurately the location of the connector but also increased the corresponding peak signature compared to what the standard OMTDR produce.

\section{STAte OF ThE ART: ON-LINE Diagnosis BASED ON REFLECTOMETRY}

Ensuring the reliable use of cables requires the availability of techniques capable of detecting the presence of faults that could potentially put in jeopardy a whole system [14]. While several electric and nonelectric wire diagnosis methods have been studied and developed, reflectometry-based techniques are still in the center stage of research and industrial applications in this domain. Reflectometry is based on the same principles as radar: a low-voltage test signal is injected down a cable where reflection occurs once an IDis is encountered. The analysis of the obtained signal permits to characterize the detected faults (type, position and characteristics). This information is very rich and valuable for maintenance operations and health monitoring of electrical networks. Notably, the main difference between reflectometry methods lies on the injected signal type, the reflected signal processing techniques and most importantly the mode of application (offline or online).

For safety critical systems, it may be necessary to monitor the health of the cables during their normal operation. Online diagnosis is then used, requiring harmlessness: diagnosis signals must not interfere with communication signals, and useful signals must not trigger any false alarm. Specific reflectometry methods have been designed for this purpose among which lie the Multi-Carrier reflectometry and the Orthogonal Multi-tone Time Domain Reflectometry (OMTDR) techniques. Notably, they showed very good potential for the diagnosis of live wire networks by taking benefit of the multi-carrier signals' capacity to control the signal bandwidth. To better understand the paper scope, we are going to recall the basic principles that control the functionality of any reflectometry method.
Once the multi-carrier signal $x(t)$ is generated, it is injected into the NUT identified by its impulse response $h(t)$ in the continuous domain where it propagates through the network and reflects back to the injection point at each IDis. The reflected multi-carrier signal $y(t)$ is thus obtained as:

$$
y(t)=x(t) * h(t)+n(t)
$$

where $n(t)$ is the channel noise. For a transmission line of length $l$, the frequency domain transfer function $H(f)$ is the inverse Fast Fourier Transform (IFFT) of $h(t)$ and is expressed as follows:

$$
H(f)=\Gamma_{E}+\frac{\left(1-\Gamma_{E}^{2}\right) \Gamma_{L} e^{-2 \gamma l}}{1+\Gamma_{E} \Gamma_{L} e^{-2 \gamma l}}
$$

where $\gamma$ is the cable's propagation constant. The reflection coefficient $\Gamma_{E}$ at the entrance of the transmission line is determined by the source impedance $Z_{0}$ and the characteristic impedance of the cable $Z_{c}$ as follows:

$$
\Gamma_{E}=\frac{Z_{c}-Z_{0}}{Z_{c}+Z_{0}}
$$

Whereas, the reflection coefficient $\Gamma_{L}$ of a load at the end of the transmission line is determined by the load impedance $Z_{L}$ and $Z_{c}$. The reflection coefficient $\Gamma_{L}=1$ in the case of an open circuit $\left(Z_{L}=\infty\right)$ while $\Gamma_{L}=-1$ in the case of a short circuit $\left(Z_{L}=0\right)$. The characteristic impedance $Z_{c}$ can be expressed as follows:

$$
Z_{c}=\sqrt{\frac{R+j \omega L}{G+j \omega C}}
$$

where $\omega=2 \pi f$ with $f$ being the frequency. The transmission line is characterized by its primary parameters $R, L, C$ and $G$ representing the resistance, inductance, capacitance and conductance per unit length. Significantly, these parameters may be theoretically calculated according to the physical and electrical proprieties of the transmission line such as conductor diameter, material, stranding, etc [15], [16]. Otherwise, they may be estimated based on the channel response measurement [17], [18]. In our case study, the exact properties of the transmission line are not essential. In fact, the physical properties are rarely divulged by the cable manufacturers and are generally protected by standards hardly accessible by large public. The propagation constant $\gamma$ in eq. (2) is expressed as follows:

$$
\gamma=\sqrt{(R+j \omega L)(G+j \omega C)}=\alpha+j \beta
$$

where, $\alpha$ is the line attenuation and $\beta$ is the phase constant. The attenuation in $(\mathrm{dB} / \mathrm{m})$ is given as:

$$
\alpha=\operatorname{Re}(\gamma)\left(\frac{20}{\log (10)}\right)
$$

Indeed, the attenuation of the signal is mainly due to the resistance of the cable: it reduces the amplitude of the signal during the propagation and therefore limits the performance 
of the diagnosis. This phenomenon is mainly encountered on long cables, and becomes more important in the presence of connectors dedicated for connecting different sections of the cable.

At the receiver end, cross-correlation is applied between the injected signal $X(f)=\operatorname{FFT}\{x(t)\}$ and the received one $Y(f)=\operatorname{FFT}\{y(t)\}$ as follows:

$$
\Gamma_{x y}(f)=Y(f) X^{*}(f)
$$

where $X^{*}(f)$ being the conjugate of $X(f)$. Performing the inverse FFT on $\Gamma_{x y}(f)$ permits to return the reflectometry response of the NUT.

As a matter of fact, MCR and its variant OMTDR have shown promising and efficient potential in detecting and locating hard and soft faults in an online live manner [3], [5], [6]. However, they suffered from signal loss related to wiring system's attenuation, coupling and complex wiring topology. For illustration purposes, Fig. 1 (top) shows the peak amplitude of an open circuit at the end of a tested Un-Shielded Twisted Pair (USTP) DRB EN 2267-010 DRB 22 for different lengths ranging from $1 \mathrm{~m}$ to $100 \mathrm{~m}$ after being injected by an OMTDR based signal composed of $N=128$ subcarriers over a total bandwidth $(\mathrm{BW})$ ranging from $300 \mathrm{KHz}$ to $1.5 \mathrm{GHz}$ with $N=2048$ samples. The amplitude has been normalized relative to the peak amplitude corresponding to the cable mismatch. Here, $Z_{c}=102 \Omega$ and $\alpha=-4 \mathrm{~dB} / \mathrm{m}$ at a frequency $f=1 \mathrm{GHz}$ based on the method of determining lineal parameters of a transmission line described in [17]. It can be noted that the amplitude decreases with an increase in the cable length. For extended lengths $L \geqslant 80 \mathrm{~m}$, the peak amplitude is very low (i.e. $\leq 0.05$ ) as shown in Fig. 1 (bottom).
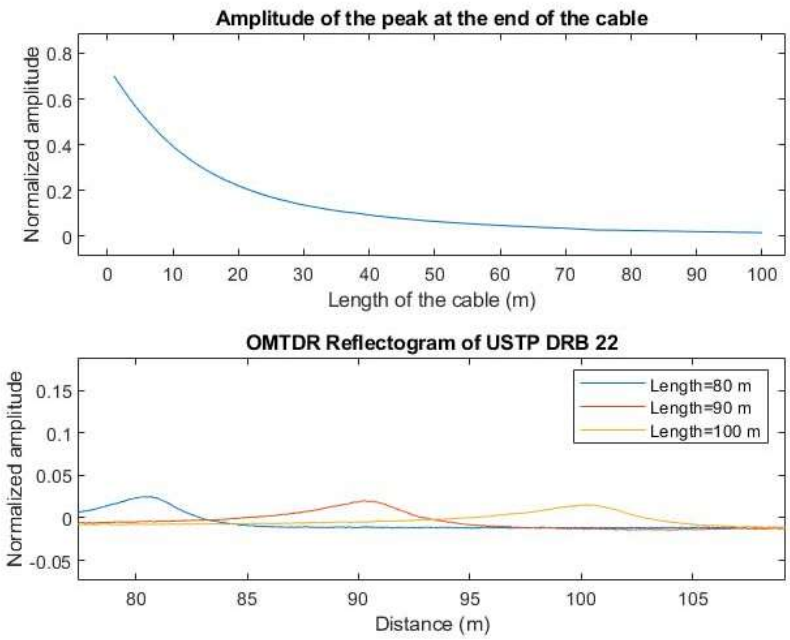

Fig. 1: Peak amplitude attenuation in terms of cable length (use case: USTP EN 2267-010 DRB 22)

\section{The TIMe ReVersal Multi-CARRIER REFLECTOMETRY (TRMCR)}

The basic principle of TR is presented in Fig 2. A transducer captures all the response of a medium from a source, and re-emits the time reversed version of this response into the propagation medium. The signal propagates back and focuses at the initial source taking benefit of the invariance property of the propagation equation with respect to the temporal variable $(t)$. It is noteworthy that the original transmitted pulse is coherently focused, both temporally and spatially, at the source's position.

In cable diagnosis, a fault localization method in a network of energy cables based on the principle of TR has been proposed in [20]. The method consists in recording a signal generated by an intermittent fault that propagates to a measurement point, reverse the measurement temporally, inject it back into the NUT and finally measure the reflected signal. The proposed method has been adapted to intermittent faults that spontaneously generate a shock wave but not to passive permanent faults, as soft ones (arcs, chafing, insulation damage, etc.). Moreover, it could not be applied on active live networks.

TR was then transposed to the problem of soft fault detection and location [9], [21], [22]. In [21], the proposed method consisted of injecting a reference signal into a NUT, measuring its echo and then temporally reversing this echo to re-inject it back into the network. Although, efficient results were obtained, it required a measurement of both the faulty network and a healthy (without fault) version of it. Besides, it has proven to be inadequate for online network diagnosis as the signals injected into the NUT can disturb the nominal operation of the cables by generating interference.

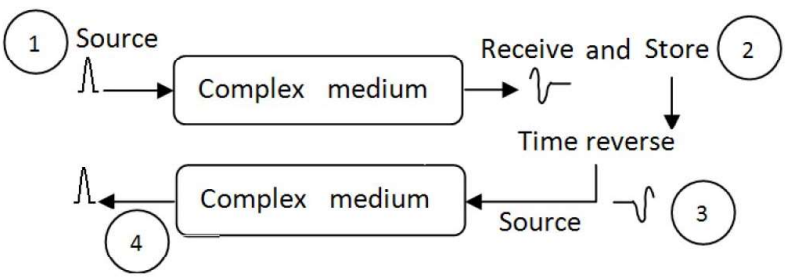

Fig. 2: A schematic explaining the TR principle in 4 steps: 1) A source generates a signal which propagates through a complex medium. 2) The received signal is stored. 3) The received signal is reversed and re-radiated into the complex medium. 4) A space time focusing at the original source is observed [19].

To cope with the aforementioned problems, TR will be applied to the multi-carrier signals of the MCR method thus enabling not only online reflectometry-diagnosis but also sensors communication in complex wiring networks. For clarification purposes, the diagram of Fig. 3 summarizes all the steps followed in the proposed algorithm.

\section{A. Generation and injection of the multi-carrier signal}

The very first phase of the TRMCR method commences by displacing switch 1 to point $\mathrm{A}$ so as to connect the signal 


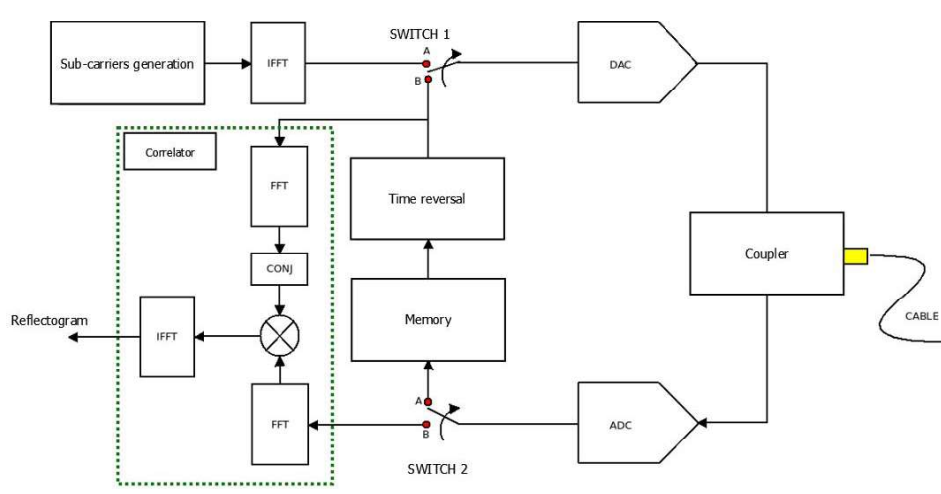

Fig. 3: Block diagram of the TRMCR method.

generator to the digital-to-analog converter (DAC) as shown in Fig. 3. The multi-carrier signal consists in dividing the bandwidth $B$ using $N$ sub-carriers. After data generation, each sub-carrier is modulated independently by a digital modulation (i.e. Quadrature Amplitude Modulation (QAM), Phase-shift keying (PSK), etc.). This modulation uses a finite number of distinct signals to represent the digital data. As an example, PSK uses a finite number of phases, each assigned a unique pattern of binary digits. Usually, each phase encodes an equal number of bits. Here, each pattern of bits forms the symbol that is represented by the particular phase and/or amplitude. In frequency domain, the obtained symbols are denoted as $\left\{X_{0}, X_{1}, \cdots, X_{N-1}\right\}$ corresponding to the frequencies $\left\{f_{0}, f_{1}, \cdots, f_{N-1}\right\}$. After that, an IFFT is applied to transform the multi-carrier signals into the discrete time domain given as $\left\{x_{0}, x_{1}, \cdots, x_{N-1}\right\}=\operatorname{ifft}\left\{X_{0}, X_{1}, \cdots, X_{N-1}\right\}$. The test signal is then defined as follows:

$$
x_{n}=\sum_{n=0}^{N-1} X_{n} e^{j 2 \pi n \Delta f\left(\frac{n T_{s}}{N}\right)}, \forall n=\{0, \cdots, N-1\} .
$$

where $T_{s}$ is the signal duration and $\Delta f$ is the frequency spacing between two consecutive sub-carriers. After applying a digital to analog conversion using a DAC, the test signal denoted as $x(t)$ is injected down the NUT having an impulse response $h(t)$. The $M$ sub-carriers in the prohibited bands are not allocated to the diagnosis system and are set to zeros to enable on-line diagnosis.

\section{$B$. Recording and time reversing the reflected multi-carrier signal}

The injected multi-carrier signal will propagate through the NUT and would reflect back to the injection point at each impedance discontinuity. Accordingly, the reflected multicarrier signal $y_{1}(t)$ is obtained according to eq. 1 as $y_{1}(t)=$ $x(t) * h(t)+n_{1}(t)$ with $n_{1}(t)$ being the channel noise.

As shown in the diagram of Fig. 3, the system includes an analog to digital converter (ADC) which handles the digitization process of the measured reflected signal $y_{1}(t)$ to $y_{1}(n)$ where $n \in\{0,1, \cdots, N-1\}$. A memory is then used for saving $y_{1}(n)$. In this phase of operation, the second switch
2 is displaced from B to A so as to connect the output of the ADC to the memory as depicted in Fig. 3.

After that, the time reversal signal processing procedure is executed. The obtained reflected signal $y_{1}(t)$ is numerically reversed in time producing $y_{1}\left(T_{s}-t\right)$ followed by saving it in the memory.

C. Numerical re-injection of the TR MC signal and recording of the reflected one

At this stage of the system operation, switch 1 is displaced from A to B in order to connect the memory to the DAC. The time reversed multi-carrier signal $y_{1}\left(T_{s}-t\right)$ is numerically re-injected into a cloned model of the NUT having the same impulse response $h(t)$ as the tested one. $y_{1}\left(T_{s}-t\right)$ will propagate through the NUT and reflects back to the injection point at each IDis. The obtained reflected signal $y_{2}(t)$ is given as follows:

$$
y_{2}(t)=y_{1}\left(T_{s}-t\right) * h(t)+n_{2}(t),
$$

given that $n_{2}(t)$ is the channel noise.

\section{Correlation pf the reflected signals}

The last step is dedicated to construct the final reflectometry response of the tested NUT where a cross-correlation is applied between the signal $Y_{1}(f)=\operatorname{FFT}\left\{y_{1}(t)\right\}$ and received one $Y_{2}(f)=\operatorname{FFT}\left\{y_{2}(t)\right\}$ as follows:

$$
\Gamma_{y_{1} y_{2}}(f)=Y_{2}(f) Y_{1}^{*}(f),
$$

where $Y_{1}^{*}(f)$ is the conjugate of $Y(f)$. Performing the inverse FFT on $\Gamma_{y_{1} y_{2}}(f)$ permits to return the reflectometry response of the NUT noted $\Gamma_{y_{1} y_{2}}(n)$.

\section{Results AND Discussions}

In order to check the applicability of the proposed technique, we opted to test the algorithm described in III numerically first and then against experimental data.

\section{A. Numerical Validation}

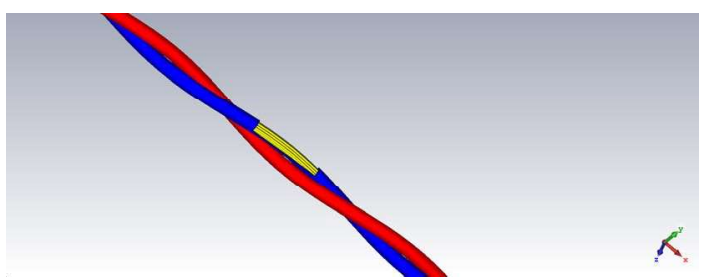

Fig. 4: A capacitive fault inserted at $10 \mathrm{~m}$ of an open circuited USTP EN 2267-010 DRB 22 cable.

In the first phase of numerical validation, we considered an open circuited point to point cable containing a $2 \mathrm{~cm}$-length capacitive fault as shown in Fig. 4 at $10 \mathrm{~m}$ from the injection point.

A transmission line Matlab-based simulation model implementing a FDTD-ABCD method formed a suitable solution for the analysis of NUTs. The impulse response of the used 


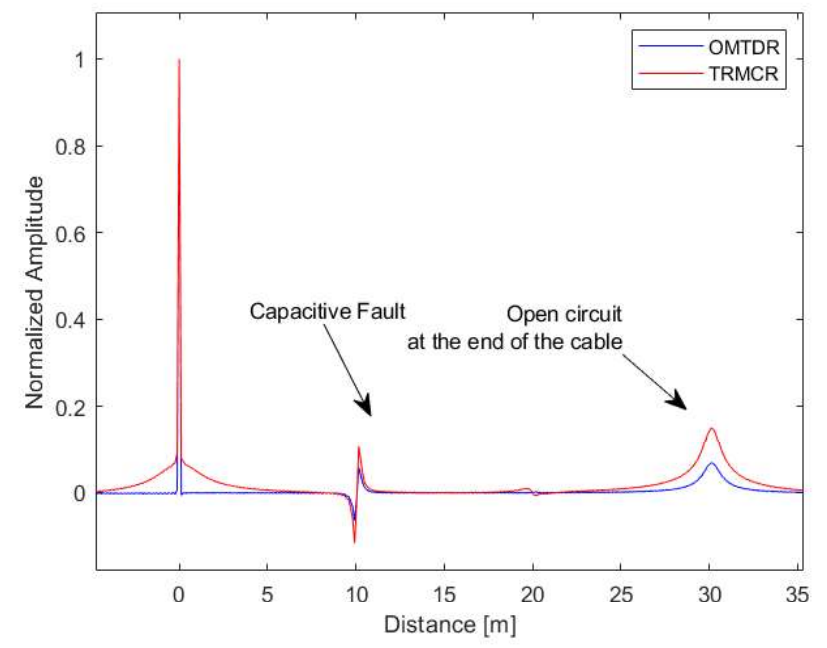

Fig. 5: Comparison of OMTDR and TRMCR results on an USTP EN 2267-010 DRB 22 with a capacitive fault

unshielded twisted pair (USTP) EN 2267-010 DRB 22 cable was computed by modeling its corresponding RLCG parameters estimated based on the method described in [17]. Besides, based on the eqs. (4) and (5), the characteristic impedance of the cable $Z_{c}=102 \Omega$ while the attenuation $\alpha=-4 \mathrm{~dB} / \mathrm{m}$.

A multi-carrier signal in addition to an OMTDR signal composed of $N=1280$ sub-carriers over a total bandwidth (BW) ranging from $\mathrm{DC}$ to $1 \mathrm{GHz}$ are numerically generated and injected into the cable under test. The standard OMTDR algorithm and the TRMCR procedure described in sec. III are applied. Fig. 5 demonstrates the obtained reflectograms for both the OMTDR (blue) and the TRMCR (red) methods. It can be clearly noticed that the TRMCR has significantly increased the soft fault peak's signature with a factor of 2 compared to the conventional multi-carrier reflectometry (OMTDR).

In a second scenario, we considered a more complex NUT as those used by the Airbus aerospace company for Helicopter cable configurations. The network is composed of 11 branches of STP EN 2714-013 MLB 22 cables having a characteristic impedance $Z_{c}=56 \Omega$ which are connected by 5 junctions. The same aforementioned process is repeated to compare the performance acquired with the proposed TRMCR method to that of standard MCR techniques manifested by the OMTDR method with an increased network complexity. Notably, it can be deduced from the obtained reflectogram of Fig. 6 that the TRMCR has significantly increased the junctions $(1 \& 2)$ signature compared to the OMTDR results. More importantly, the same achievement is prevailed even on junction 3 that is $10 \mathrm{~m}$ away from the injection point although this kind of cables suffer from high attenuation $(11 \mathrm{~dB} / \mathrm{m}$ at $1 \mathrm{GHz})$. Accordingly, TRMCR is well suited for the diagnosis of networks composed from such high attenuation cables and complexified multiple junction NUTs.

Numerical results have been extended to a $100 \mathrm{~m}$ coaxial EN 4604-003 WZ cable $\left(Z_{c}=50 \Omega\right.$ and $\left.\alpha=-0.4 \mathrm{~dB} / \mathrm{m}\right)$ with

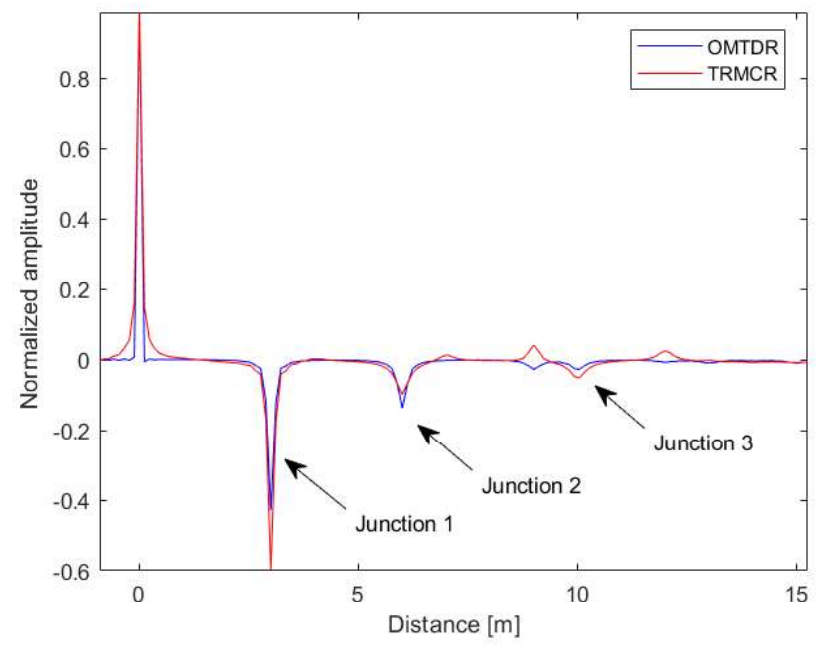

Fig. 6: Comparison of OMTDR and TRMCR results on a complex NUT composed of 11 branches connected by 5 junctions.

aeronautical D38999 Series connectors as that demonstrated in Fig. 9 at each $25 \mathrm{~m}$ (airplane configuration) of the line. The objective is to study and evaluate the response of the connector signature depending on the length and complexity of the network. To numerically model the impulse response of the connector, its scattering parameters were measured by a Vector Network Analyzer (VNA) on frequency bandwidth from $9 \mathrm{KHz}$ to $2 \mathrm{GHz}$ over 10000 samples. Since our simulation model is based on the ABCD matrix due to its easy cascading properties making it possible to take into account changes in the line by cascading matrices of different line sections, the connector's $S$ parameters are converted into $\mathrm{ABCD}$ matrix form according to [23].

The OMTDR and TRMCR algorithms are then applied on this NUT based on a multi-carrier signal with $N=1280$ subcarriers over a total $\mathrm{BW}$ ranging from $\mathrm{DC}$ to $1 \mathrm{GHz}$. The FM band [87.5 MHz-108 MHz] is considered as the prohibited band and the corresponding samples from 439 to 541 are set to zeros. The obtained reflectometry responses are plotted in Fig.7 where it can be observed that TRMCR has remarkably increased the connectors' signatures thus improving their visibility compared to what the standard OMTDR provides. It is noteworthy that appearance of secondary lobes on the final results as shown in Fig.7 can be returned to the unknowable NUT response in the prohibited bandwidth. Significantly, the numerical results have proven the usefulness and effectiveness of the TRMCR method in lively improving the signature of impedance discontinuities in different complexity wire networks.

\section{B. Experimental Validation}

For an additional feasibility proof, experimental tests were conducted on a $35 \mathrm{~m}$ long STP EN 2714-013 MLB 22 with a D38999 connector placed at $30 \mathrm{~m}$ from the injection point in order to serve as a real-life demonstration for the performance 


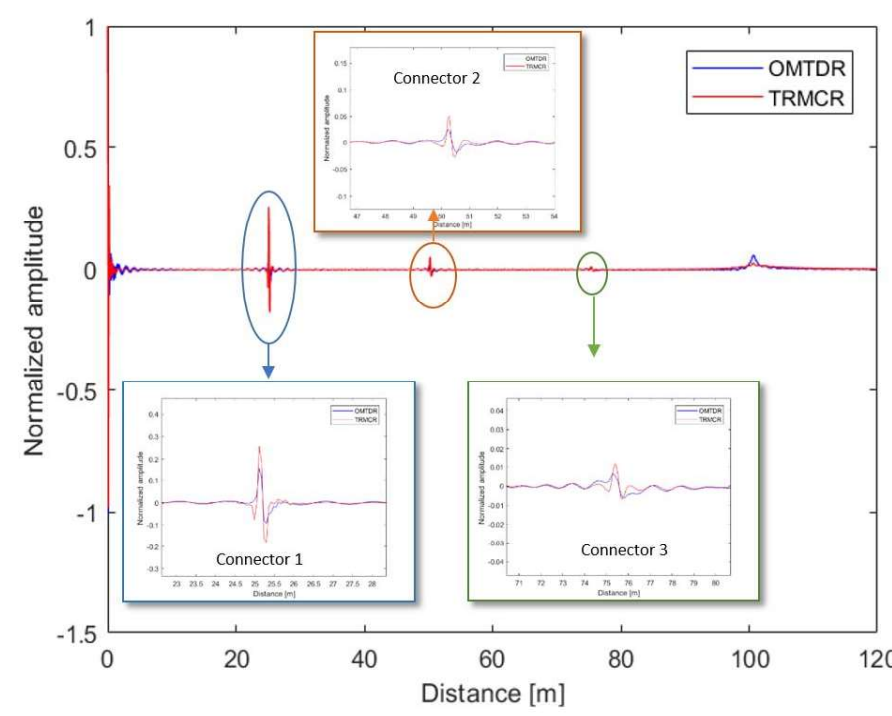

Fig. 7: Comparison of OMTDR and TRMCR results on an airplane NUT (connector at each $25 \mathrm{~m}$ ).

of the proposed approach. The process of generation, injection and acquisition of multi-carrier signals into a NUT was possible by a specific architecture that has been developed and designed at our lab.

In this architecture, the MCR/OMTDR-based reflectometry was integrated in a Field-Programmable Gate Array: FPGA SoC Xilinx ZyNQ 7010, 10-bits ADC, 10-bits DAC and 512 Megabyte DDR chip as an electronic board under the name of "Ariane V2" as that presented in Fig.8. The injection/acquisition procedure to collect the MCR/OMTDR response is accomplished by a software developed in Python. This program performs the MCR/OMTDR-based communication via a Computer Unit (CU) by either a wired or a wireless link. On the other hand, the data processing and the TR steps of the TRMCR method detailed in sec. III are accomplished using a post-processing Matlab based software on a standard state-of-the art computer.

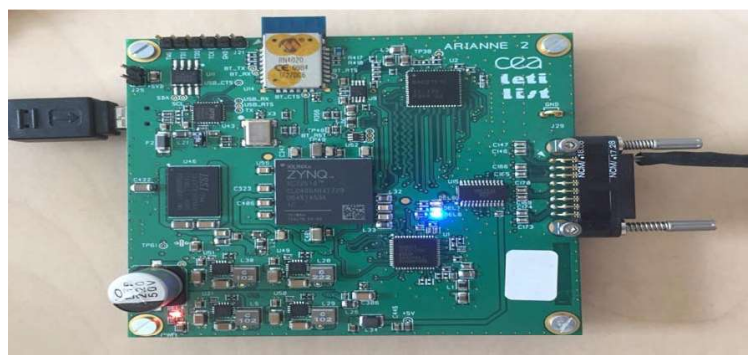

Fig. 8: The "Ariane V2" electronic board used for generating, injecting and receiving the MCR/OMTDR signals into a tested wiring network.

Accordingly, a multi-carrier OMTDR signal composed of $N=128$ sub-carriers over a total bandwidth ranging from $300 \mathrm{KHz}$ to to $1.5 \mathrm{GHz}$ with 2048 frequency samples has been generated and injected into the tested network. Sig- nificantly, TRMCR not only located accurately the location of the connector but also increased the corresponding peak signature compared to what the standard OMTDR produced as demonstrated in Fig. 9.

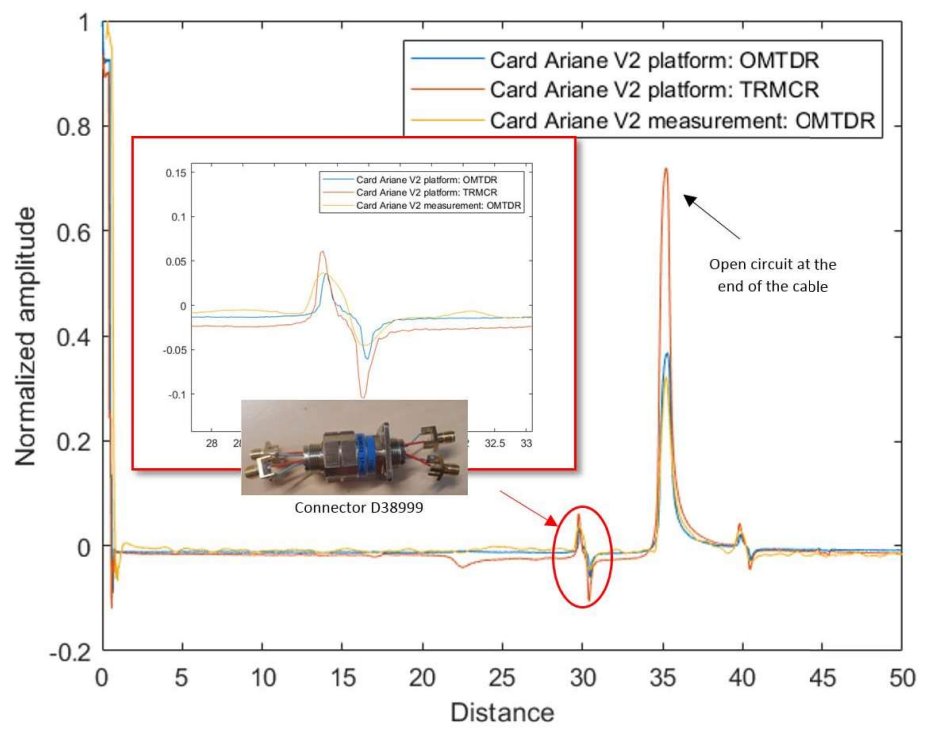

Fig. 9: Experimental results on a STP EN 2714-013 MLB 22 cable in the presence of a D38999 connector at $30 \mathrm{~m}$ from the injection port.

\section{CONCLUSION}

This paper has investigated the performance of TRMCR method for online network diagnosis and sensor communication in complex wiring networks. To do so, a single testing extremity is required thus reducing the diagnosis system's complexity (no synchronization, simple implementation, possible boarding, etc.).

Numerical simulation-based tests performed on a wide range of cables (unshielded/shielded twisted pair, coaxial cable, etc.) along with practical-application networks (Helicopters/airplane configuration) have validated the efficiency of TRMCR in detecting and locating impedance discontinuities while the NUT is in normal operation. The most interesting feature is the method's ability to increase the IDis's peak signatures thus enhancing their detectability making them more tolerant to noise compared to what the standard MCR methods provide. Experimental results supported the aforementioned claims and asserted the feasibility of the proposed approach in increasing the detection gain and hence improving the diagnosis coverage. The increased complexity of the NUT has proven to positively enhance the performance of the TRMCR method, as an increasingly longer sequence of echoes systematically leads to a higher fraction of energy being concentrated into the peak echo.

\section{REFERENCES}

[1] P. Smith, C. Furse, and J. Gunther, "Analysis of spread spectrum time domain reflectometry for wire fault location," IEEE sensors journal, vol. 5, no. 6, pp. 1469-1478, 2005. 
[2] C. Furse, P. Smith, C. Lo, Y. C. Chung, P. Pendayala, and K. Nagoti, "Spread spectrum sensors for critical fault location on live wire networks," Structural Control and Health Monitoring: The Official Journal of the International Association for Structural Control and Monitoring and of the European Association for the Control of Structures, vol. 12, no. 3-4, pp. 257-267, 2005.

[3] S. Naik, C. M. Furse, and B. Farhang-Boroujeny, "Multicarrier reflectometry," IEEE Sensors Journal, vol. 6, no. 3, pp. 812-818, 2006.

[4] L. Incarbone, F. Auzanneau, W. B. Hassen, and Y. Bonhomme, "Embedded wire diagnosis sensor for intermittent fault location," in SENSORS, 2014 IEEE. IEEE, 2014, pp. 562-565.

[5] W. B. Hassen, F. Auzanneau, L. Incarbone, F. Pérès, and A. P. Tchangani, "On-line diagnosis using orthogonal multi-tone time domain reflectometry in a lossy cable," in Proceedings of the 10th International Multi-Conference on Systems, Signals and Devices (SSDâĂŹ13), 2013, pp. 1-6.

[6] L. Incarbone, S. Evain, W. B. Hassen, F. Auzanneau, A. Dupret, Y. Bonhomme, F. Morel, R. Gabet, L. Solange, and A. Zanchetta, "Omtdr based integrated cable health monitoring system smartco: An embedded reflectometry system to ensure harness auto-test," in Industrial Electronics and Applications (ICIEA), 2015 IEEE 10th Conference on. IEEE, 2015, pp. 1761-1765.

[7] M. Fink, "Time reversal of ultrasonic fields. i. basic principles," IEEE transactions on ultrasonics, ferroelectrics, and frequency control, vol. 39, no. 5, pp. 555-566, 1992.

[8] F. Wu, J.-L. Thomas, and M. Fink, "Time reversal of ultrasonic fields. il. experimental results," IEEE transactions on ultrasonics, ferroelectrics, and frequency control, vol. 39, no. 5, pp. 567-578, 1992.

[9] L. Abboud, A. Cozza, and L. Pichon, "A matched-pulse approach for soft-fault detection in complex wire networks," IEEE Transactions on Instrumentation and Measurement, vol. 61, no. 6, pp. 1719-1732, 2012.

[10] L. Abboud, A. Cozza, and L. Pichon, "A noniterative method for locating soft faults in complex wire networks," Vehicular Technology, IEEE Transactions on, vol. 62, no. 3, pp. 1010-1019, 2013.

[11] M. Kafal, A. Cozza, and L. Pichon, "Locating faults with high resolution using single-frequency tr-music processing," IEEE Transactions on Instrumentation and Measurement, vol. 65, no. 10, pp. 2342-2348, 2016.

[12] M. Kafal, J. Benoit, A. Cozza, and L. Pichon, "A statistical study of dort method for locating soft faults in complex wire networks," IEEE Transactions on Magnetics, vol. 54, no. 3, pp. 1-4, 2018.

[13] M. Kafal, J. Benoit, A. Cozza, and L. Pichon, "Soft fault diagnosis in wire networks using time reversal concept and subspace methods," Proc. EEETEM.

[14] F. Auzanneau, "Wire troubleshooting and diagnosis: Review and perspectives," Progress In Electromagnetics Research, vol. 49, pp. 253-279, 2013.

[15] Q. Shi, U. Tröltzsch, and O. Kanoun, "Analysis of the parameters of a lossy coaxial cable for cable fault location," in Systems, Signals and Devices (SSD), 2011 8th International Multi-Conference on. IEEE, 2011, pp. 1-6.

[16] T. K. Truong, "Twisted-pair transmission-line distributed parameters," EDN Mag., 2000.

[17] J. Cohen and N. Gregis, "Method of determining lineal parameters of a transmission line," Patent US20 170269 137A1, 2014.

[18] X. Jiao and Y. Liao, "A linear estimator for transmission line parameters based on distributed parameter line model," in Power and Energy Conference at Illinois (PECI), 2017 IEEE. IEEE, 2017, pp. 1-8.

[19] M. Kafal, "Techniques d'imagerie pour la détection et la localisation de défauts non francs dans les réseaux de câblage," Ph.D. dissertation, Université Paris-Saclay, 2016.

[20] M. Paolone, F. Rachidi-Haeri, H. Mahmoudimanesh, R. Razzaghi, and G. Lugrin, "Efficient method based on the electromagnetic time reversal to locate faults in power network," Tech. Rep., 2014.

[21] L. El Sahmarany, L. Berry, N. Ravot, F. Auzanneau, and P. Bonnet, "Time reversal for soft faults diagnosis in wire networks," Progress In Electromagnetics Research, vol. 31, pp. 45-58, 2013.

[22] M. Kafal, A. Cozza, and L. Pichon, "Locating multiple soft faults in wire networks using an alternative dort implementation," Instrumentation and Measurement, IEEE Transactions on, vol. 65, no. 2, pp. 399-406, 2016.

[23] D. A. Frickey, "Conversions between s, z, y, h, abcd, and t parameters which are valid for complex source and load impedances," IEEE Transactions on microwave theory and techniques, vol. 42, no. 2, pp. 205-211, 1994. 